Argonne National Laboratory ("Argonne") under Contract No. W-31-109-ENG-38 with the U.S. Department of Energy. The U.S.

Government retains for itself, and others act-

ing on its behalf, a paid-up, nonexclusive,

irrevocable worldwide license in said article

to reproduce, prepare derivative works, dis-

tribute copies to the public, and perform pub-

licly and display publicly, by or on behalf of

the Government.

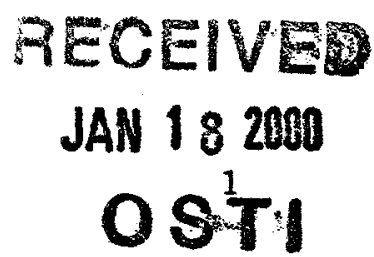

\title{
Tevatron Direct Photon Results
}

\section{S. Kuhlmann ${ }^{\text {a }}$}

Argonne National Laboratory

Tevatron direct photon results since DISS8 are reviewed. Two new CDF measurements are discussed, the Run Ib inclusive photon cross section and the photon +muon cross section. Comparisons with the latest NLO QCD calculations are presented.

\section{Inclusive Photons}

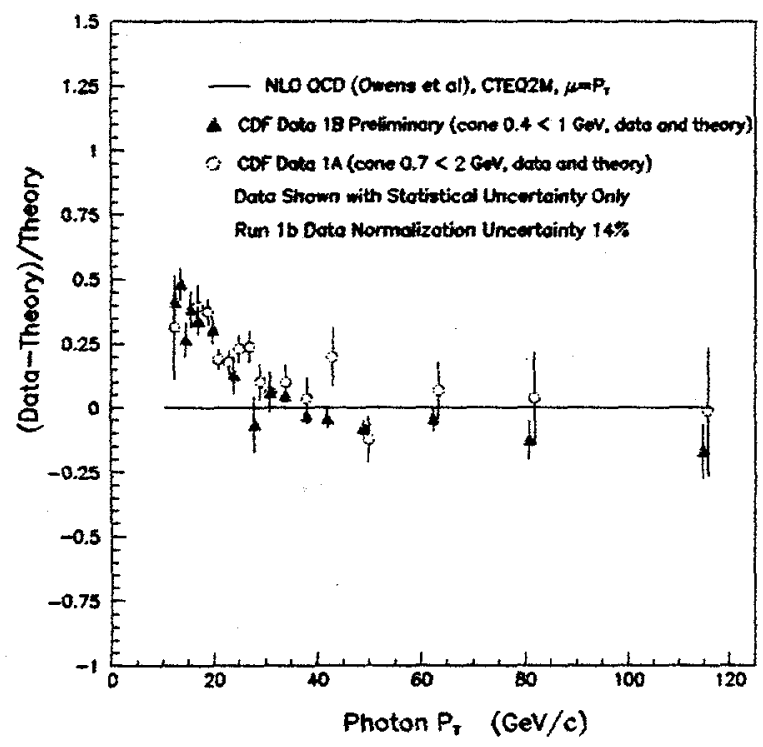

Figure 1. Run 1a and Run 1b isolated photon measurements from CDF, compared to the theory used in the Run la publication. (see text)

The Run 1a measurement of direct photons from CDF [1] difficult to explain with NLO QCD calculations [2,3]. One hypothesis was that additional Kt was needed, which would cause an increasing deviation from NLO QCD as Pt was decreased. Therefore the best measurement at the lowest Pt possible was needed. The data be low $16 \mathrm{GeV}$ in the Run $1 \mathrm{a}$ measurement suffered from low statistics, as shown in fig. 1. A change in the Run Ib trigger thresold has improved statistics in this region by $x 20$. This measurement is also shown in fig. 1. The two measurements are consistent, with the improved bins below $16 \mathrm{GeV}$ showing the largest deviation from the QCD calculation.

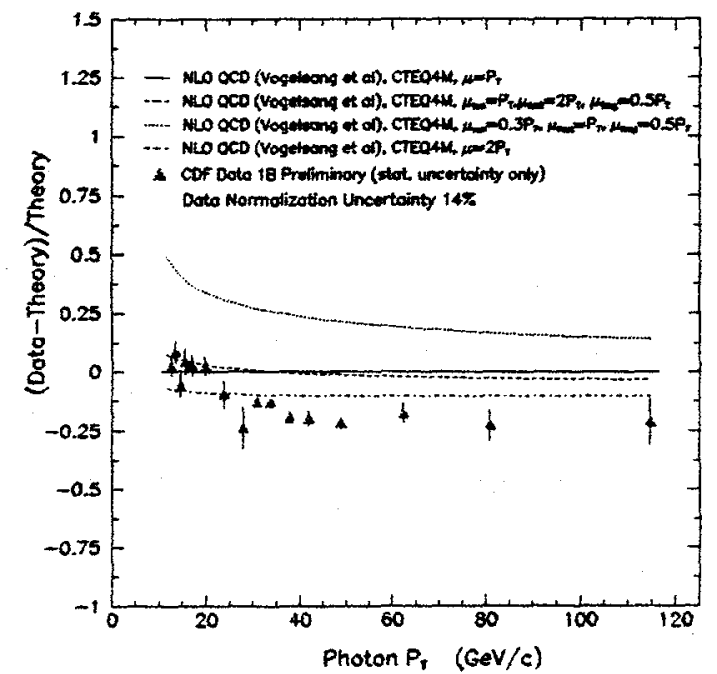

Figure 2. Comperison of CDF Run $1 b$ inclusive photons with the latest QCD predictions (see text).

* Work supported by the U.S. Department

of Energy, Division of High Energy Physics Contract W-31-109-ENG-38. 


\section{DISCLAIMER}

This report was prepared as an account of work sponsored by an agency of the United States Government. Neither the United States Government nor any agency thereof, nor any of their employees, make any warranty, express or implied, or assumes any legal liability or responsibility for the accuracy, completeness, or usefulness of any information, apparatus, product, or process disclosed, or represents that its use would not infringe privately owned rights. Reference herein to any specific commercial product, process, or service by trade name, trademark, manufacturer, or otherwise does not necessarily constitute or imply its endorsement, recommendation, or favoring by the United States Government or any agency thereof. The views and opinions of authors expressed herein do not necessarily state or reflect those of the United States Government or any agency thereof. 


\section{DISCLAIMER}

Portions of this document may be illegible in electronic image products. Images are produced from the best available original document. 
The QCD calculation shown in fig. 1 is somewhat outdated, and is only shown here for historical reasons. The calculation shown in fig. 2 uses modern parton distributions, includes the NLO fragmentation piece, and is shown using a considerable range of scale choices. Of course not every combination of scales and parton distributions can be shown, but in general no combination has been found that matches the shape of the data to within several standard deviations.

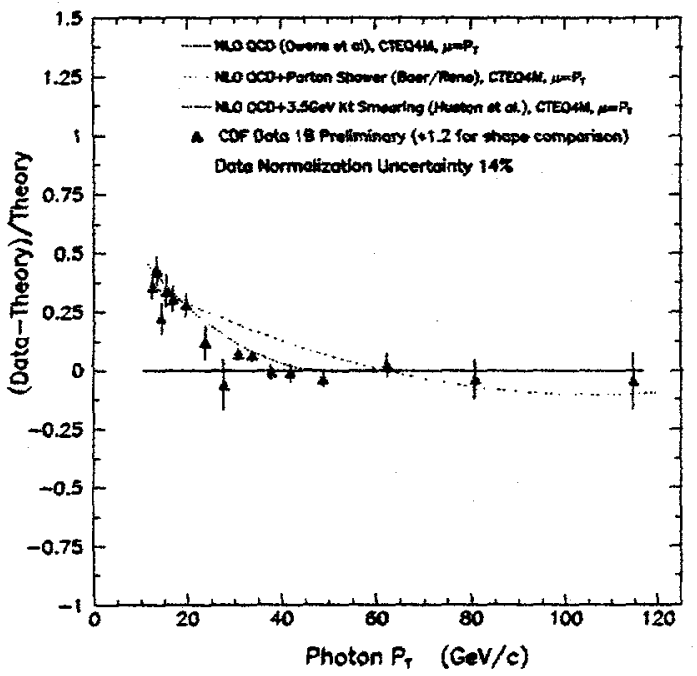

Figure 3. Comparison of CDF Run $1 b$ inclusive photons with two calculations that add $\mathrm{Kt}$ to NLO QCD. (see text)

$=$

One possibility that does match the data is adding $\mathrm{Kt}$ to the NLO QCD calculation. We use the exact same procedure that E706 uses in their analysis [4], except with a $\mathrm{Kt}$ value of 3.5 $\mathrm{GeV}$. This is not intrinsic Kt, but the part of the non-collinear initial state radiation that might be missing in NLO QCD. Fig. 3 shows that this works very well in representing the shape of the data, when all the other theory parameters are set to "typical" values. This is of course an ad- hoc procedure, the other curve shown in fig. 3 is the first attempt by Baer-Reno to do a more theoretical approach by adding a parton shower to NLO QCD. Further refinements of this procedure are underway, as well 88 analytic resummations of soft gluons.

\section{Photon + Muon Production}

The same dataset used for the inclusive photon cross section in the last section can be used for more exclusive studies. In this case CDF looks for muon candidates with cuts typical of many other CDF analyses involving muons. The number of candidates are then compared with the expected backgrounds from charged pion and kaon decay-in-flight and punchthrough, this is shown in fig. 4. An excess of events is seen above these backgrounds.

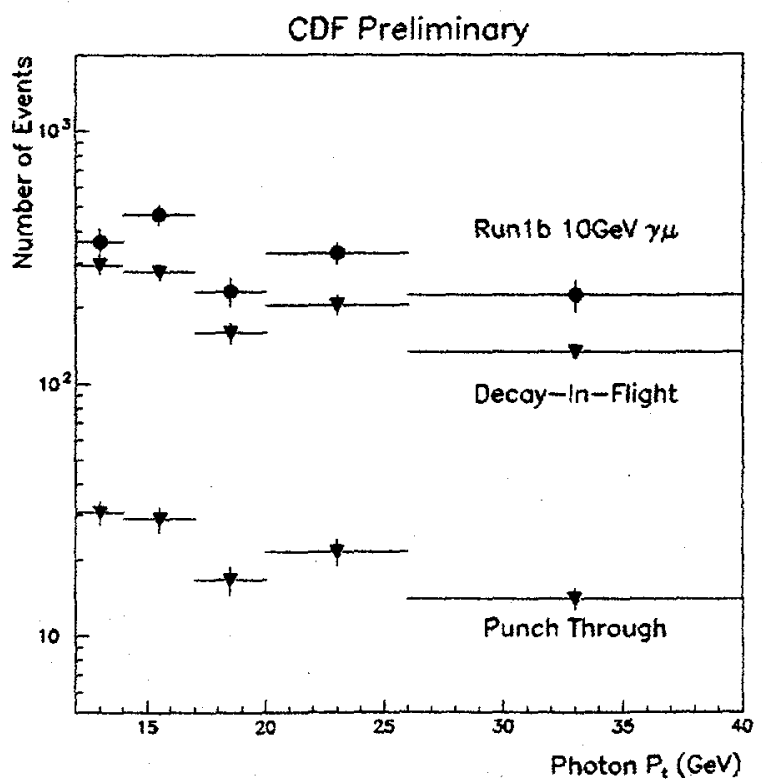

Figure 4. Photon + muon production at CDF is compared to the expected backgrounds from muons from pion and kaon decays and punchthrough. 
Fortunately QCD predicts an additional source of muons from photon+charm and photon+bottom production. The leading order diagram is compton scattering a gluon off a heavy quark in the proton, giving rise to a photon plus the heavy quark. The heavy quark then decays semi-leptonically into a muon. This measurement is compared with calculations from PYTHIA and NLO QCD. The PYTHIA curves do not contain the time-consuming bremsstrahlung diagram, which is known to be a substantial fraction of the cross section, hence this comparison is a bit misleading. The NLO QCD calculation [5], which does include this diagram, is in decent agreement with the data. Note that in this case we are cutting on the Pt of objects on both sides of the event, hence the effects of $\mathrm{Kt}$ smearing should be reduced. Also note that in both calculations the fraction of cross section coming from bottom decays is surprisingly large, of order $30 \%$. This comes from the harder decay spectrum of the muon coming from bottom decays, as opposed to charm decays.

\section{Conclusions}

Direct photon production continues to be a good place to test modern NLO QCD calculations. The inclusive photons continue to be plagued with shape differences between data and theory. Inclusion of Kt smearing effects in simple gaussian smearing models works well, but for gluon distribution studies one needs a more fundamental approach such as resummation.

\section{REFERENCES}

1. F. Äbe et al. (CDF Collaboration). Phys. Rev. Lett 73, 2662 (1994).

2. H. Baer, J. Ohnemus, and J.F. Owens. Phys. Rev. D42, 61 (1990).

3. M. Gluck et al. Phys. Rev. Lett. 73, 388 (1994).

4. L. Apanasevich et al. Phys. Rev. D59, 74007 (1999).

5. B. Bailey, E. Berger, L. Gordon. Phys. Rev. D54, 1896 (1996).

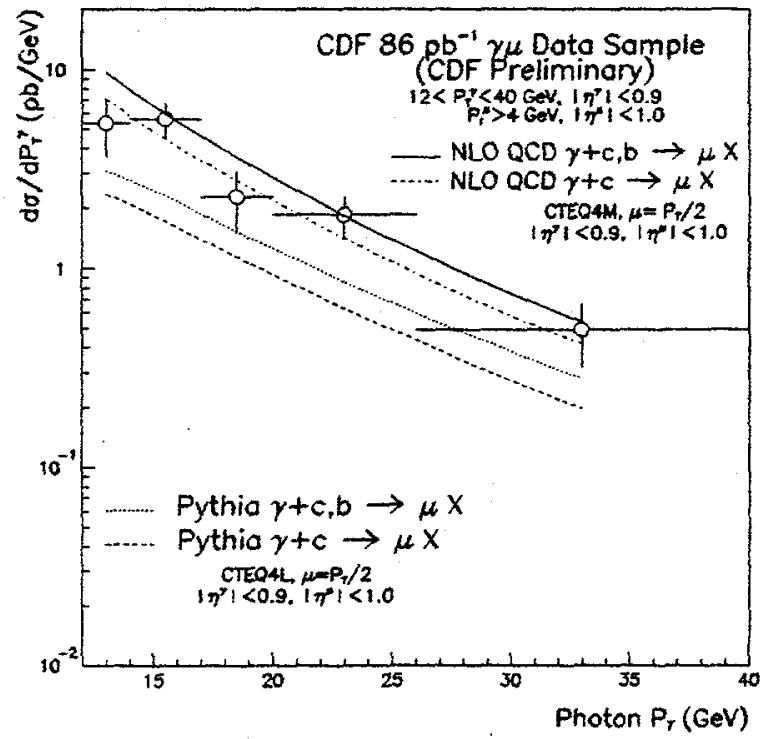

Figure 5. Photon + muon production from CDF is compared to NLO QCD calculations, as well as the PYTHIA monte carlo. 